\title{
Klotho Ameliorates Kidney Injury and Fibrosis and Normalizes Blood Pressure by Targeting the Renin-Angiotensin System
}

\author{
Lili Zhou, ${ }^{* \dagger}$ Hongyan Mo, ${ }^{*}$ Jinhua Miao, ${ }^{*}$ Dong Zhou, ${ }^{\dagger}$ Roderick J. Tan, ${ }^{\dagger}$ Fan Fan Hou, ${ }^{*}$ and Youhua Liu*
}

Accepted for publication August 27, 2015.

Address correspondence to Youhua Liu, Ph.D., Department of Pathology, University of Pittsburgh School of Medicine, S-405 Biomedical Science Tower, 200 Lothrop St., Pittsburgh, PA 15261. E-mail: liuy@upmc.edu.

\begin{abstract}
Loss of Klotho and activation of the renin-angiotensin system (RAS) are common pathological findings in chronic kidney diseases. However, whether these two events are intricately connected is poorly understood. We hypothesized that Klotho might protect kidneys by targeted inhibition of RAS activation in diseased kidneys. To test this hypothesis, mouse models of remnant kidney, as well as adriamycin nephropathy and unilateral ureteral obstruction, were utilized. At 6 weeks after 5/6 nephrectomy, kidney injury was evident, characterized by elevated albuminuria and serum creatinine levels, and excessive deposition of interstitial matrix proteins. These lesions were accompanied by loss of renal Klotho expression, up-regulation of RAS components, and development of hypertension. In vivo expression of exogenous Klotho through hydrodynamic-based gene delivery abolished the induction of multiple RAS proteins, including angiotensinogen, renin, angiotensin-converting enzyme, and angiotensin II type 1 receptor, and normalized blood pressure. Klotho also inhibited $\beta$-catenin activation and ameliorated renal fibrotic lesions. Similar results were obtained in mouse models of adriamycin and obstructive nephropathy. In cultured kidney tubular epithelial cells, Klotho dose-dependently blocked Wnt1-triggered RAS activation. Taken together, these results demonstrate that Klotho exerts its renal protection by targeted inhibition of RAS, a pathogenic pathway known to play a key role in the evolution and progression of hypertension and chronic kidney disorders. (Am J Pathol 2015, 185: 3211-3223; http://dx.doi.org/10.1016/j.ajpath.2015.08.004)
\end{abstract}

Chronic kidney disease (CKD) is increasingly recognized as a major public health problem, because it affects about $10 \%$ to $13 \%$ of the adult population worldwide. ${ }^{1-3}$ Among many risk factors for developing CKD, aging is an independent and strong predictor for progressive renal insufficiency. ${ }^{4}$ The elderly population also tends to develop hypertension and cardiovascular disease, characteristic features consistent with the activation of the renin-angiotensin system (RAS). ${ }^{5,6}$ These observations suggest that aging, CKD, and RAS activation might be intimately linked. However, the molecular details behind these connections are not fully understood.

RAS consists of several key components, including angiotensinogen (AGT), renin, angiotensin-converting enzyme (ACE), and angiotensin II type I and type II receptors (AT1 and AT2, respectively). ${ }^{7-9}$ Two main enzymes in this system, renin and ACE, lead to the formation of angiotensin II, the principal active peptide of RAS, which mediates both blood pressure-dependent and -independent kidney damage in CKD. Several factors can induce RAS activation, such as reactive oxygen species, hyperglycemia, and albumin. ${ }^{10,11} \mathrm{We}$ recently found that all RAS genes contain T cell factor/ lymphoid enhancer-binding factor binding sites in their promoter regions and are directly regulated by canonical Wnt/ $\beta$-catenin signaling. ${ }^{12}$ These results have established that $\beta$ catenin is a master regulator controlling the expression of all RAS components in the kidneys.

Supported by National Natural Science Foundation of China grants 81130011 and 81521003 (Y.L.) and 81370014 (L.Z.); NIH grants DK064005, DK091239, and DK106049 (Y.L.); grant LYM10043 from the Foundation for Distinguished Young Talents in Higher Education of Guangdong, China (L.Z.); and grant sybzzxm201223 from the Foundation for Excellent Doctoral Dissertation of Guangdong, China (L.Z.).

Disclosures: None declared. 

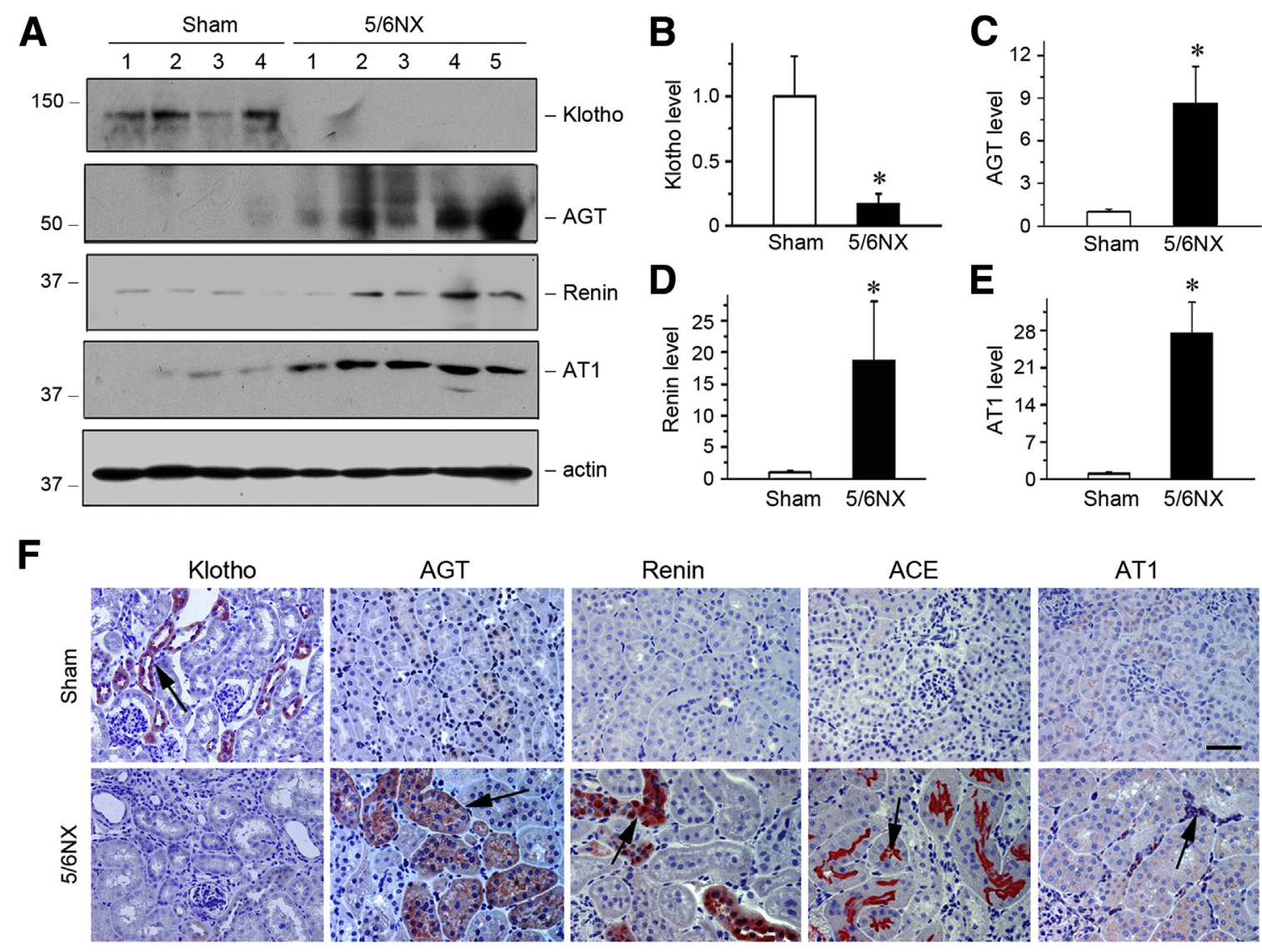

Figure 1 Repression of Klotho is associated with renal renin-angiotensin system (RAS) activation in a mouse remnant kidney model. A: Western blot analyses of renal expression of Klotho and RAS components in sham control and 5/6 nephrectomized mice (5/6NX). Kidney lysates from sham control and 5/ $6 \mathrm{NX}$ mice at 6 weeks as indicated were immunoblotted with antibodies against Klotho, AGT, renin, AT1, and actin, respectively. B-E: Graphic presentation of the relative abundance of Klotho (B), AGT (C), renin (D) and AT1 (E) in two groups as indicated. F: Representative micrographs show Klotho, AGT, renin, ACE, and AT1 protein expression in two groups. Paraffin-embedded kidney sections were stained with different antibodies. Arrows indicate positive staining. ${ }^{*} P<0.05$ versus sham controls. $n=5$ to $6(\mathbf{B}-\mathbf{E})$. Scale bar $=50 \mu \mathrm{m}(\mathbf{F})$.

Klotho is a novel antiaging protein that is highly expressed in all major tubular segments of normal adult kidneys, and its expression is progressively diminished in aging animals and humans. ${ }^{13,14}$ Klotho is a type I single-pass transmembrane protein comprising a large extracellular region with two homologous KL1 and KL2 domains, a transmembrane segment, and a short intracellular domain. ${ }^{15}$ Besides this fulllength membranous form, alternative splicing and/or proteolytic shedding of its extracellular region also generates a short soluble and secreted form. We have previously shown that both membranous and soluble Klotho physically bind to multiple Wnt ligands such as Wnt1, Wnt4, and Wnt7a, all of which are up-regulated in injured kidneys. ${ }^{16}$ This Klotho/Wnt interaction results in functional sequestration of Wnt ligands, and blocks Wnt-mediated $\beta$-catenin activation and its target gene expression. Therefore, loss of Klotho, which is common in aged kidneys and $\mathrm{CKD},{ }^{13,17}$ will inevitably lead to $\mathrm{Wnt} / \beta$-catenin activation and development of kidney lesions and renal insufficiency.

Both Klotho depletion and RAS activation are common pathological findings in various CKD with different etiologies. However, whether, and how, these two events are connected is poorly understood. Because Klotho inhibits Wnt/ $\beta$-catenin, ${ }^{16}$ a signaling pathway that controls the expression of multiple RAS genes, ${ }^{12}$ we hypothesized that Klotho may protect kidneys by targeted inhibition of RAS activation. Here, we have systematically investigated the regulation of RAS proteins by Klotho in three models of CKD induced by 5/6 nephrectomy (5/6NX), adriamycin, or unilateral ureteral obstruction (UUO), respectively. Using an in vitro model, we have also demonstrated that Klotho is able to abolish the induction of multiple RAS proteins in cultured kidney tubular epithelial cells. Our results establish that the antiaging protein Klotho exerts its renal protection by inhibition of RAS, a pathogenic pathway that plays a pivotal role not only in the pathogenesis of CKD but also in the development of hypertension and cardiovascular disorders.

\section{Materials and Methods}

\section{Animal Models}

All animal experiments were approved the Laboratory Animal Committee at the Southern Medical University and the Institutional Animal Care and Use Committee at the University of 

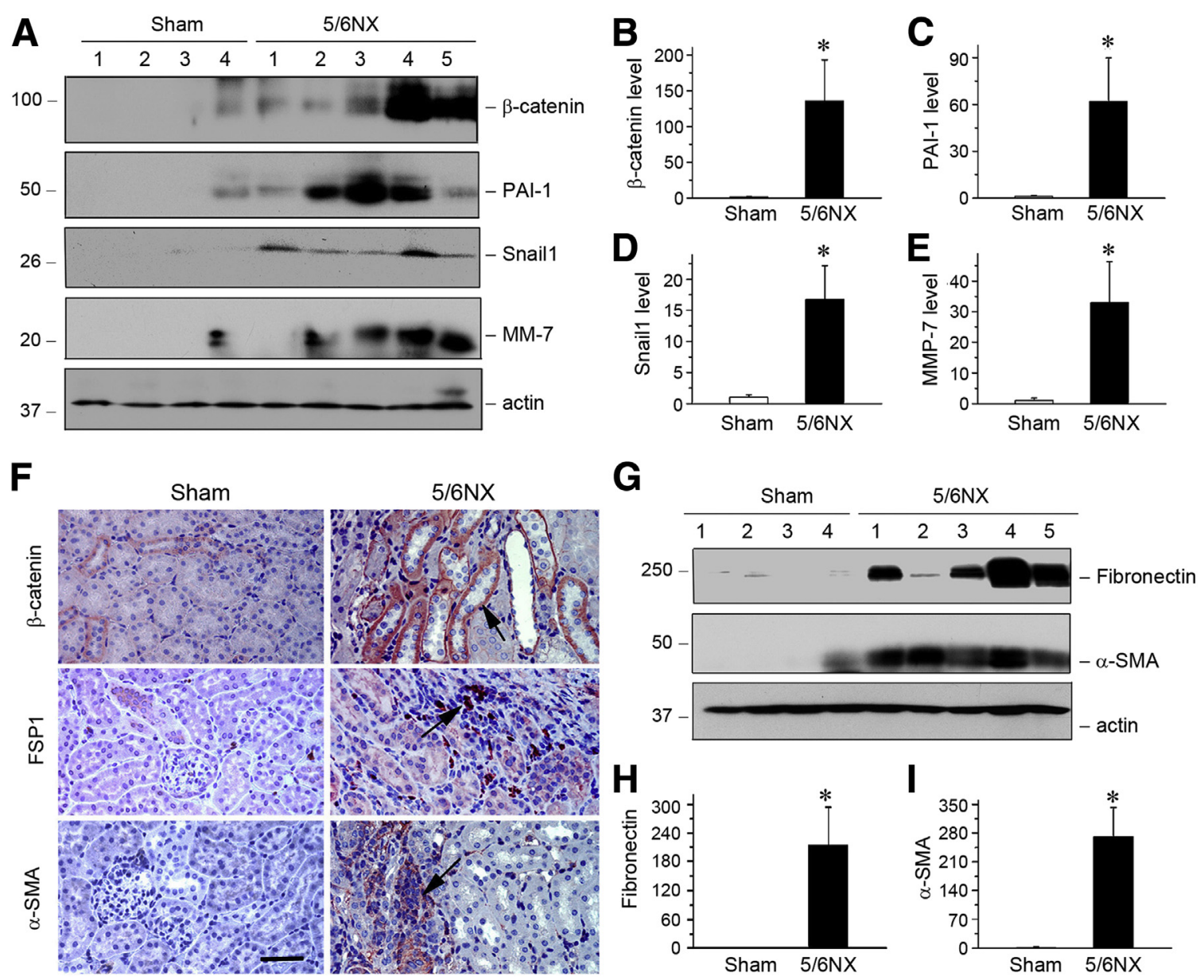

Figure 2 Expression of $\beta$-catenin and its target genes in the mouse remnant kidney model. A: Representative Western blot analyses show $\beta$-catenin and its target proteins in kidney lysates. Whole kidneys were homogenized and blotted with antibodies against $\beta$-catenin and its targets plasminogen activator inhibitor-1 (PAI-1), Snail1, and matrix metalloproteinase (MMP)-7. Actin was used as a loading control. B-E: Quantitative analyses demonstrate the overexpression of $\beta$-catenin (B), PAI-1 (C), Snail1 (D), and MMP-7 (E) in 5/6NX mice. F: Representative micrographs show $\beta$-catenin and FSP1 and $\alpha$-SMA expression in two groups as indicated. Kidney sections were stained with different antibodies against $\beta$-catenin, FSP1, and $\alpha$-SMA. Arrows indicate positive staining. G-I: Western blot analyses show the expression of fibronectin and $\alpha$-SMA in different groups as indicated. Western blot $(\mathbf{G})$ and graphical presentation of fibronectin $(\mathbf{H})$ and $\alpha$-SMA $(\mathbf{I}) . n=5$ to 6 (B-E, $\mathbf{H}$, and $\mathbf{I})$. ${ }^{*} P<0.05$ versus sham controls. Scale bar $=50 \mu \mathrm{m}(\mathbf{F})$.

Pittsburgh, respectively. Animal experiments were performed in compliance with the National Institute of Health's Guidelines for the Care and Use of Laboratory Animals. For the 5/6NX model, male CD-1 mice, weighing approximately 23 to $25 \mathrm{~g}$, were subjected to the surgical resection of the upper and lower poles, two thirds of the left kidney, or sham operation. One week later (week 0), the intact right kidney was removed via a right flank incision, as previously described. ${ }^{18,19}$ One week after the operation (week 1), the 5/6NX mice were randomized and divided into different groups ( $n=6$ to 8 in each group): i) sham control; ii) $5 / 6 \mathrm{NX}$ mice injected with empty vector pcDNA3 (designated as 5/6NX group); and iii) 5/6NX mice injected with $\mathrm{pV} 5$-sKlotho plasmid (designated as $5 / 6 \mathrm{NX}+\mathrm{K}$ group). In vivo expression of secreted Klotho in mice was performed by a hydrodynamic-based gene delivery approach, as described previously. ${ }^{16}$ At week 6, all mice were euthanized, and urine and kidney tissue were collected for various analyses. Mouse models of adriamycin nephropathy and UUO were described previously, ${ }^{15}$ and the archival kidney samples from these earlier studies were used for analyzing the expression of RAS proteins.

\section{Blood Pressure Measurements}

Tail-cuff measurements of systolic (SBP) and diastolic blood pressures were performed by a validated method that relies on volume pressure recording technology (CODA 8; Kent Scientific Corporation, Torrington, CT). This method has been validated by using a telemetry system. ${ }^{20-22}$ In brief, all mice were acclimated to the system for 5 consecutive days immediately followed by 8 days of baseline measurements. One day before the experiment was completed, tail-cuff measurements of SBP and diastolic blood pressures was performed on all mice. Data recording consisted of 5 acclimation cycles followed by 15 measurement cycles, with little time elapse between 


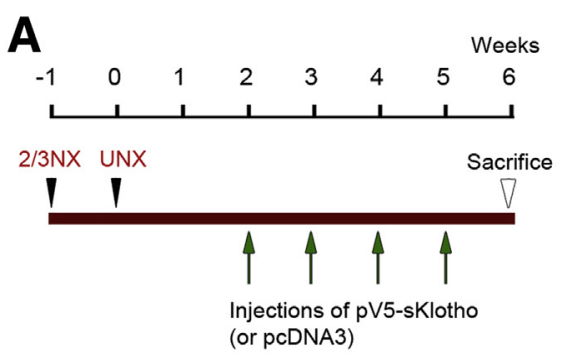

D

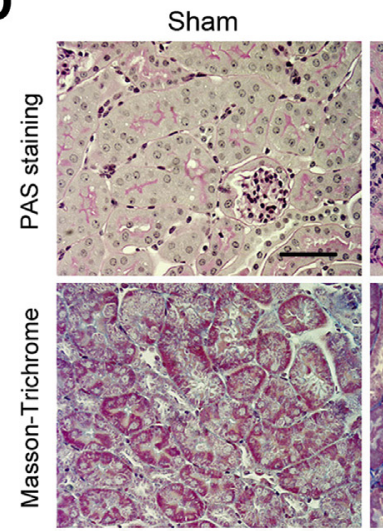

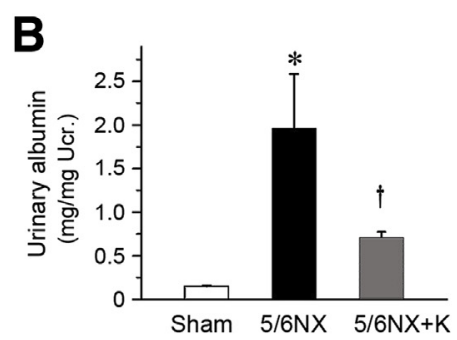

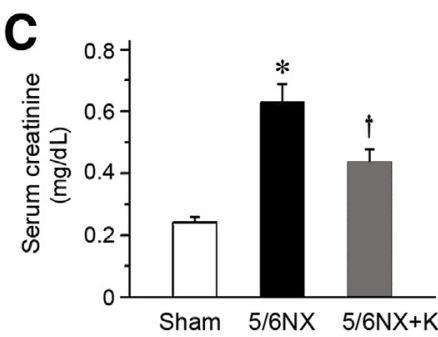

$\mathbf{E}$
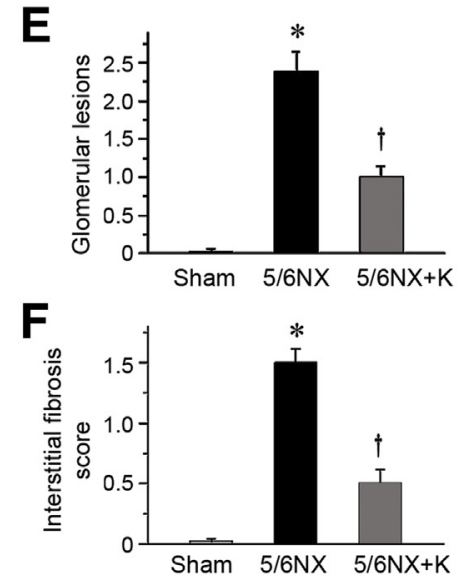

Figure 3 Expression of secreted Klotho in vivo ameliorates renal injury in mouse model of remnant kidney. A: Experimental design. Green arrows indicate the injection of pV5-sKlotho plasmid; black arrowheads, the surgical resection of left kidney and nephrectomy of right kidney. B: Urinary albumin levels in mice at 6 weeks after 5/6NX. Urinary albumin was expressed as $\mathrm{mg} / \mathrm{mg}$ creatinine. C: Klotho reduces serum creatinine levels in 5/6NX mice. Serum creatinine was assessed at 6 weeks after 5/6NX. D-F: Representative images show that expression of Klotho attenuates glomerular lesions and interstitial fibrosis as indicated by periodic acid-Schiff (PAS) and Masson's trichrome staining (D) and quantitative data of glomerular lesions (E) and interstitial fibrosis score (F) in different groups. Paraffin sections were used for periodic acid-Schiff and Masson's trichrome staining. Black arrow indicates positive staining. ${ }^{*} P<0.05$ versus sham controls; ${ }^{\dagger} P<0.05$ versus $5 / 6 \mathrm{NX}$ alone. Scale bar $=50 \mu \mathrm{m}$ (D). $2 / 3 \mathrm{NX}$, two thirds of left kidney by surgical resection; $5 / 6 \mathrm{NX}, 5 / 6$ nephrectomized mice injected with pcDNA3; 5/6NX+K,5/6 nephrectomized mice injected with pV5-sKlotho; UNX, intact nephrectomy of right kidney.

readings. Data recordings were accepted when SBP was between 80 and $200 \mathrm{~mm} \mathrm{Hg}$ and were averaged to obtain the average SBP and diastolic blood pressure.

\section{Urinary Albumin and Creatinine Assay}

Urinary albumin was measured by using a mouse Albumin ELISA Quantitation kit, according to the manufacturer's protocol (Bethyl Laboratories, Montgomery, TX). ${ }^{23,24}$ Urinary albumin was expressed as $\mathrm{mg} / \mathrm{mg}$ creatinine. Urinary and serum creatinine levels were determined by use of a QuantiChrom creatinine assay kit (DICT-500; Bioassay Systems, Hayward, $\mathrm{CA}$ ), according to the protocols specified by the manufacturer.

\section{Histology Assessment}

Paraffin-embedded mouse kidney sections (3- $\mu \mathrm{m}$ thickness) were prepared by a routine procedure. ${ }^{25}$ Sections were stained with hematoxylin and eosin, and periodic acid-Schiff reagents by standard protocol. The extent of glomerular lesions was assessed by a semiquantitative analysis using the method of Raij et $\mathrm{al}^{26}$ : grade 0 , no mesangial expansion and glomerular hypertrophy of glomerulus; grade 1 , mesangial expansion and glomerular hypertrophy of glomerulus of up to $25 \%$; grade 2 , mesangial expansion and glomerular hypertrophy of glomerulus of $25 \%$ up to $50 \%$; grade 3, mesangial expansion and glomerular hypertrophy of glomerulus of $50 \%$ up to $75 \%$; and grade 4, mesangial expansion and glomerular hypertrophy of glomerulus of $75 \%$ to $100 \%$. At least 50 glomeruli were evaluated under $\times 40$ magnification and results averaged for each kidney. The sclerosis index for each mouse was calculated as follows: $(\mathrm{N} 1 \times 1+\mathrm{N} 2 \times 2+\mathrm{N} 3 \times 3+\mathrm{N} 4 \times 4) / n$, where N1, N2, N3, and N4 represent the numbers of glomeruli that exhibited grades $1,2,3$, and 4 , respectively, and $n$ represents the number of glomeruli assessed. Kidney sections were also subjected to Masson-Trichrome staining for assessing collagen deposition and fibrotic lesions. Kidney interstitial fibrosis score were assessed according to the following scale: 0 , no evidence of interstitial fibrosis; $1,<25 \%$ involvement; $2,25 \%$ to $50 \%$ involvement; and $3,>50 \%$ involvement. ${ }^{27}$ The scale for each animal was reported as the mean of 20 random high-powered $(\times 400)$ fields per section.

\section{Immunohistochemical Staining}

Immunohistochemical staining was performed using routine protocol. $^{28}$ Antibodies used were as follows: goat polyclonal anti-Klotho (AF1819; R\&D Systems, Minneapolis, $\mathrm{MN}$ ), goat polyclonal anti-AGT (sc-7419; Santa Cruz Biotechnology, Santa Cruz, CA), goat polyclonal anti-renin 
A

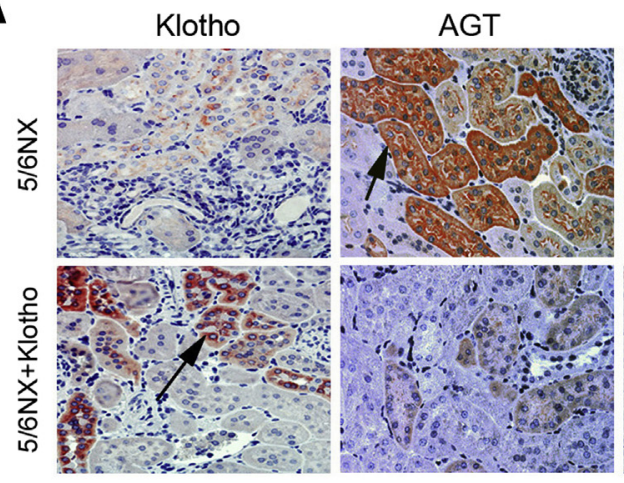

B

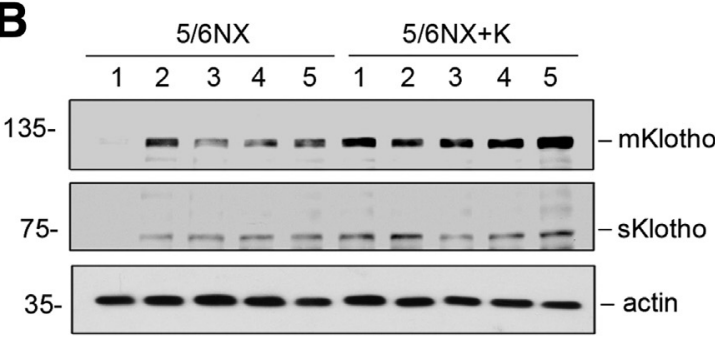

E

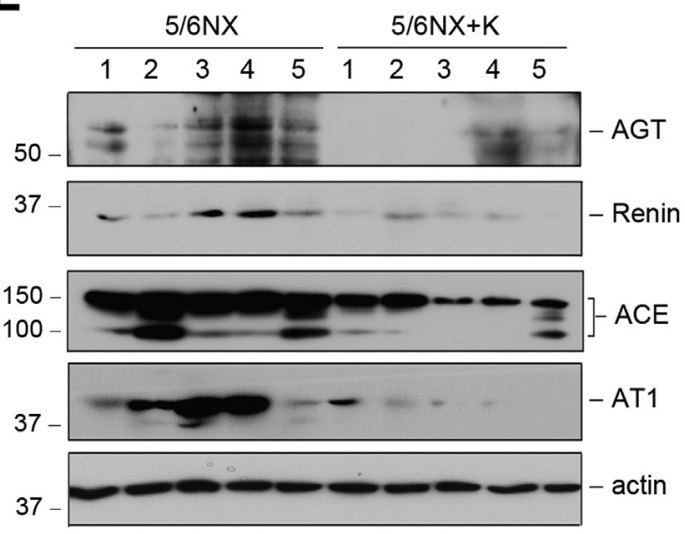

Renin

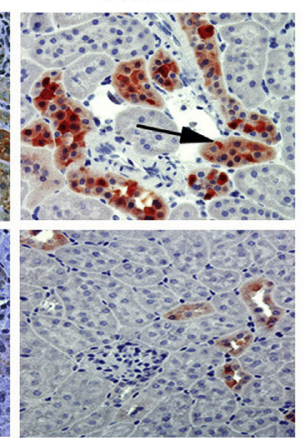

C

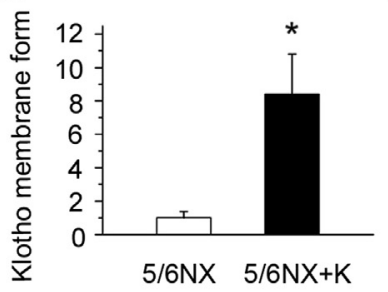

F
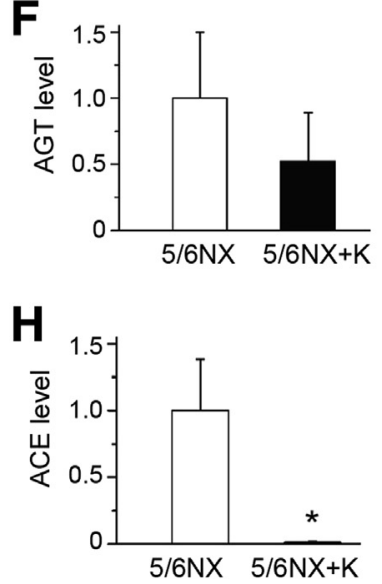

ACE

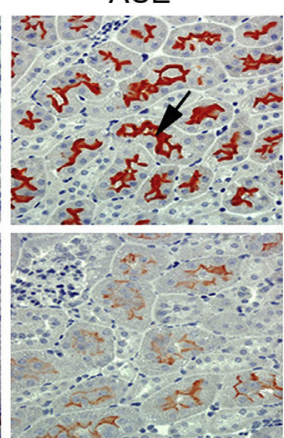

AT1

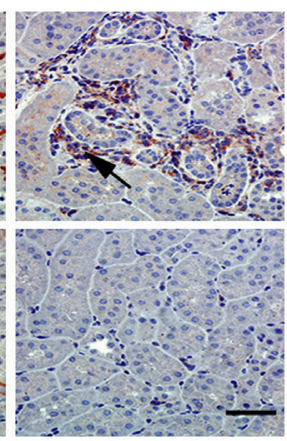

D

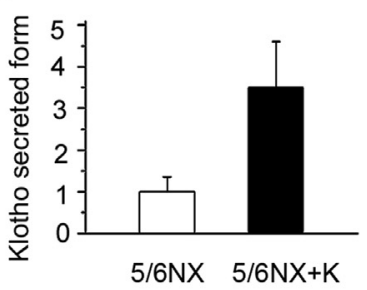

G

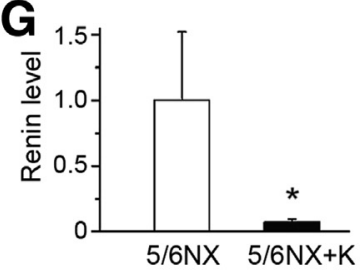

I

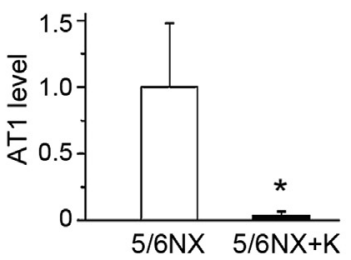

Figure 4 Expression of secreted Klotho in vivo abolishes renin-angiotensin system (RAS) induction in mouse remnant kidney model. A: Representative micrographs show Klotho and RAS components in different groups as indicated. Kidney sections were stained with different antibodies against Klotho, AGT, renin, ACE, and AT1. Arrows indicate positive staining. B: Representative Western blot analyses show membranous and secreted Klotho in kidney lysates. Renal endogenous membranous Klotho (mKlotho) and secreted Klotho (sKlotho) were indicated. Quantitative analyses demonstrate the levels of renal mKlotho (C) and sKlotho (D) at 6 weeks in 5/6NX mice (7 days after last injection of pV5-sKlotho plasmid). E: Representative Western blot analyses reveal that Klotho abolishes RAS induction in 5/6NX mice. Kidney lysates from different groups as indicated were immunoblotted with antibodies against AGT, renin, ACE, AT1, and actin, respectively. Numbers (1 to 5 ) represent different animals in a given group. $\mathbf{F}-\mathbf{I}$ : Graphic presentations of the relative abundances of AGT (F), renin $(\mathbf{G})$, ACE $(\mathbf{H})$, and AT1 (I) in different groups as indicated. ${ }^{*} P<0.05$ versus $5 / 6 \mathrm{NX}$ alone. Scale bar $=50 \mu \mathrm{m}(\mathbf{A}) .5 / 6 \mathrm{NX}, 5 / 6$ nephrectomized mice injected with pcDNA3; $5 / 6 \mathrm{NX}+\mathrm{K}, 5 / 6$ nephrectomized mice injected with pV5-sKlotho.

(sc-27320; Santa Cruz Biotechnology), rabbit monoclonal anti-ACE (ab75762; Abcam, Cambridge, MA), rabbit polyclonal anti-AT1 receptor (ab15552; Millipore, Billerica, MA), rabbit polyclonal anti- $\beta$-catenin (ab15180; Abcam), rabbit polyclonal anti-FSP-1(S100A4) (A5114; Dako, Carpinteria, CA), mouse monoclonal anti- $\alpha$-SMA (A2547; Sigma-Aldrich, St. Louis, MO), rabbit polyclonal anti-TGF$\beta 1$ (sc-146; Santa Cruz Biotechnology), and rabbit polyclonal anti-MMP-7 (GTX104658; GeneTex, Irvine, CA). After incubation with primary antibodies at $4^{\circ} \mathrm{C}$ overnight, the slides were then stained with horseradish peroxidase-conjugated secondary antibody (Jackson ImmunoResearch Laboratories,
West Grove, PA). Nonimmune normal IgG was used to replace primary antibodies as a negative control, and no staining occurred. Slides were viewed under a Nikon Eclipse E600 microscope equipped with a digital camera (Nikon, Melville, NY).

\section{Immunofluorescence Staining and Confocal Microscopy}

Kidney cryosections were fixed with $3.7 \%$ paraformaldehyde for 15 minutes at room temperature. After blocking with $10 \%$ donkey serum for 30 minutes, the slides were immunostained with primary antibodies against 

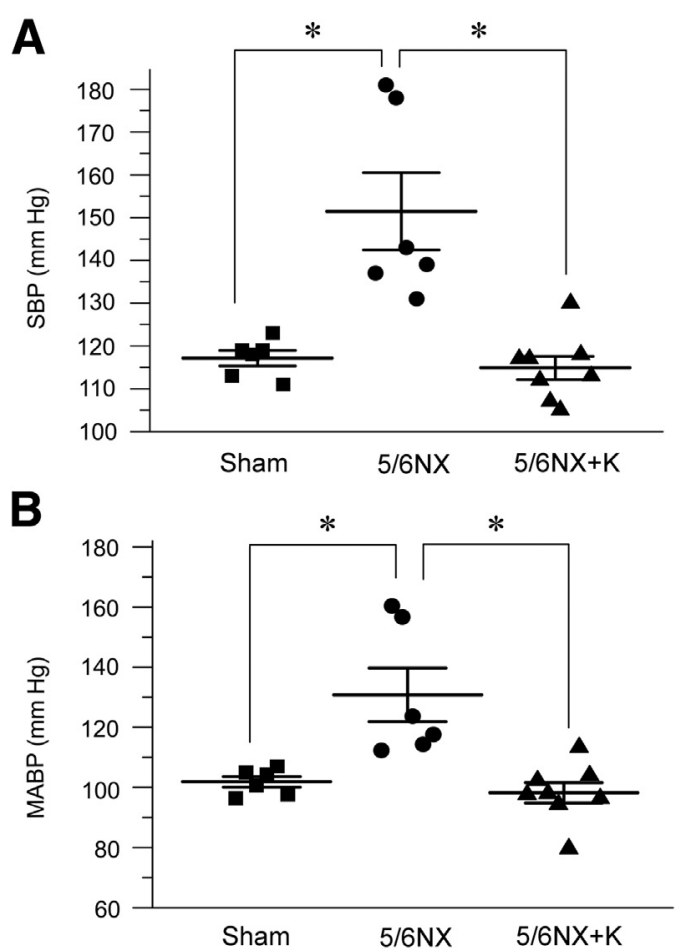

Figure 5 Expression of secreted Klotho in vivo normalizes blood pressure in 5/6NX mice. A: Tail-cuff blood pressure measurements show that delivery of pV5-sKlotho plasmid normalizes systolic blood pressure $(\mathrm{SBP})$ in mice at 6 weeks after 5/6NX. B: Exogenous sKlotho normalizes mean arterial blood pressure (MABP) at 6 weeks after $5 / 6$ NX. Solid blocks, dots, and triangles represent different animals in a given group. $n=6$ to 8 (each group). ${ }^{*} P<0.05 .5 / 6 \mathrm{NX}, 5 / 6$ nephrectomized mice injected with pcDNA3; 5/6NX+K, 5/6 nephrectomized mice injected with pV5-sKlotho.

anti-collagen I (234167; EMD Millipore), anti-AGT (Santa Cruz Biotechnology), anti-PAI-1 (sc-5297; Santa Cruz Biotechnology), and anti-fibronectin (F3648; Sigma-Aldrich). Slides were viewed under a Nikon Eclipse E600 microscope or Leica TCS-SL confocal microscope (Leica, Wetzlar, Germany) equipped with a digital camera.

\section{Western Blot Analysis}

Protein expression was analyzed by Western blot analysis as described previously. ${ }^{29}$ The primary antibodies used were as follows: anti-Klotho (R\&D System), anti-AGT (Santa Cruz Biotechnology), anti-renin (Santa Cruz Biotechnology), anti-ACE (Abcam), anti-AT1 receptor (Millipore), anti- $\beta$ catenin (610154; BD Transduction Laboratories, San Jose, CA), anti-PAI-1 (Santa Cruz Biotechnology), anti-Snail1 (ab17732; Abcam), anti-MMP-7 (3801; Cell Signaling), anti-fibronectin (Sigma-Aldrich), anti- $\alpha$-SMA (SigmaAldrich), and anti-actin (MAB1501; Millipore, Billerica, MA).

\section{Cell Culture and Treatment}

Human proximal tubular epithelial cells (HKC, clone-8) were provided by Dr. Lorraine C. Racusen (Johns Hopkins
University, Baltimore, MD). Cells were cultured according to the procedures described previously, ${ }^{12}$ and transfected with Wnt1-expressing vector or pV5-mKlotho plasmid by lipofectamine 2000 (Life Technologies, Carlsbad, CA), according to the manufacturer's protocol. Whole-cell lysates were prepared and subjected to Western blot analyses.

\section{Statistical Analysis}

All data examined were expressed as means \pm SEM. Data were analyzed using SigmaStat software version 3 (Jandel Scientific, San Rafael, CA). Comparison between groups was made by $t$-test when comparing two groups, or one-way analysis of variance followed by Student-Newman-Kuels test for more than two groups. $P<0.05$ was considered significant.

\section{Results}

Loss of Klotho Is Associated with RAS Activation in Remnant Kidneys

We first examined the expression of Klotho by Western blotting and immunostaining in remnant kidney mouse model induced by $5 / 6 \mathrm{NX}$. Klotho was markedly down-regulated in remnant kidneys at 6 weeks after 5/6NX, compared with sham controls (Figure 1, A and F). This observation, together with previous reports ${ }^{16}$ suggests that loss of antiaging protein Klotho is a common pathological finding in the pathogenesis of various CKD. We next assessed the expression of RAS components in remnant kidneys after 5/6NX. Western blotting analyses of whole-kidney homogenates demonstrated a dramatic induction of major components of RAS, such as AGT, renin, AT1 (Figure 1, A, C-E) and ACE (data not shown). Similar results were obtained when kidney tissues were examined by immunostaining (Figure 1F). The vast majority of RAS components such as AGT, renin, and ACE were predominantly induced in renal tubular epithelium (Figure $1 \mathrm{~F}$ ). These data indicate that loss of Klotho is associated with induction of RAS components in remnant kidneys, suggesting that these two events might be somehow connected in vivo.

\section{Wnt/ $\beta$-Catenin Potentially Links Klotho Depletion to RAS Activation}

Previous studies show that Klotho is an endogenous antagonist of $\mathrm{Wnt} / \beta$-catenin signaling, ${ }^{12,30}$ which controls the expression of multiple RAS components in diseased kidneys. ${ }^{12}$ Therefore, Wnt/ $\beta$-catenin might be the potential mediator that connects Klotho depletion to RAS activation in remnant kidneys after 5/6NX. To test this hypothesis, we examined the expression of $\beta$-catenin and its target proteins in this model. $\beta$-catenin protein level and its target proteins plasminogen activator inhibitor-1 (PAI-1), Snail1, and matrix metalloproteinase-7 (MMP-7) (23,28,31 $^{\text {were dramatically }}$ induced after $5 / 6 \mathrm{NX}$, as shown by Western blot analyses 
A

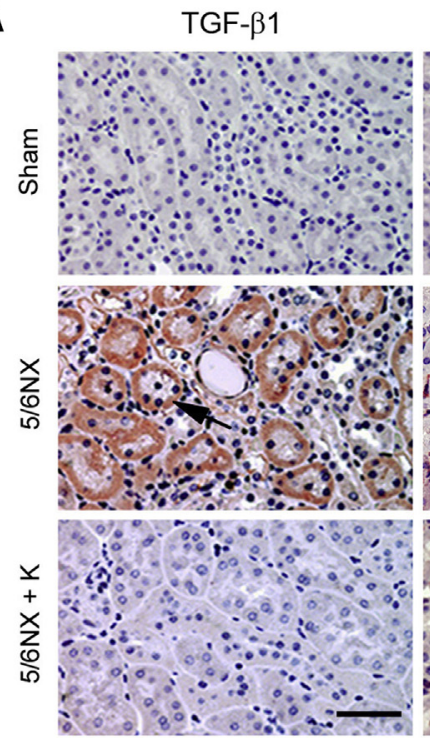

$\alpha-S M A$

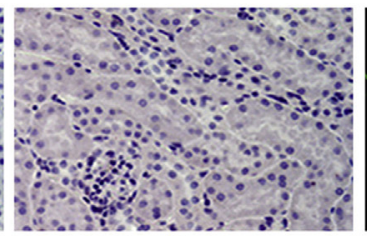

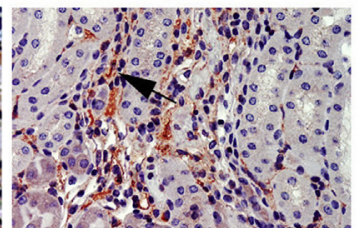

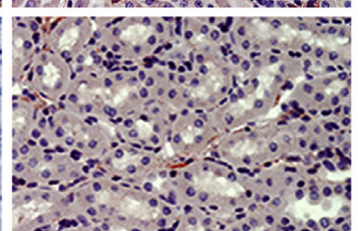

Collagen I
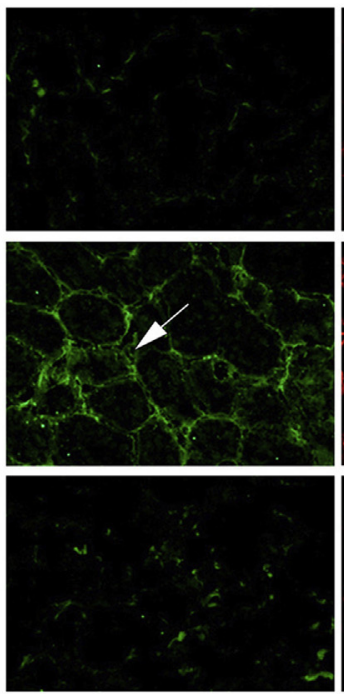

Fibronectin
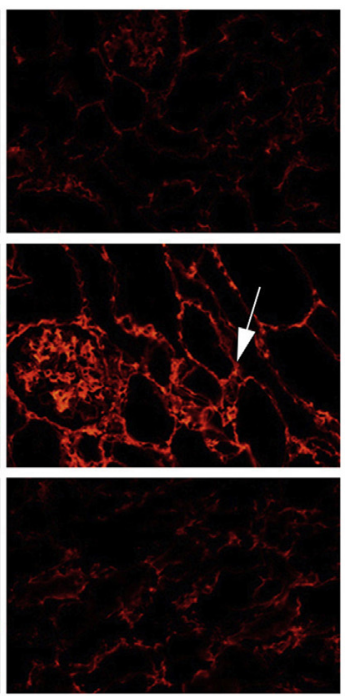

B

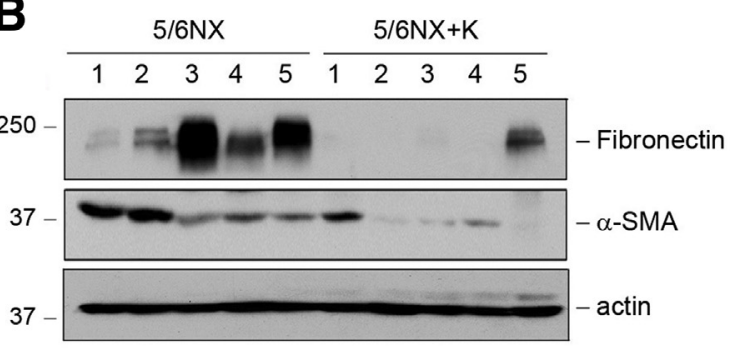

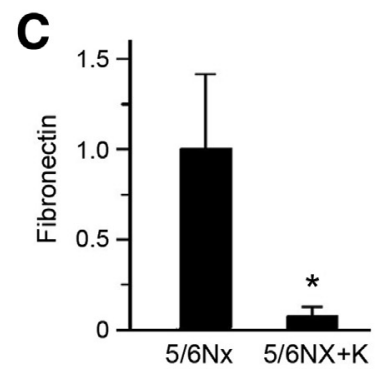

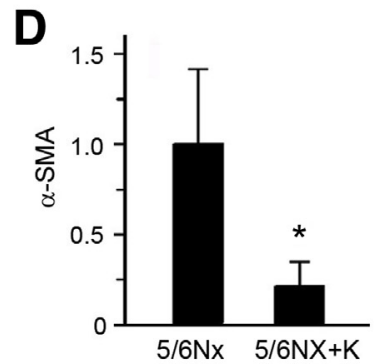

Figure 6 Klotho represses various fibrogenic genes in the mouse remnant kidney model. A: Representative micrographs show that Klotho inhibits TGF- $\beta 1$ and $\alpha$-SMA expression and reduces collagen I and fibronectin deposition in the kidneys after 5/6NX. Arrows indicate positive staining. B-D: Western blot analyses of renal expression of fibronectin and $\alpha$-SMA in 5/6NX and 5/6NX injected with pV5-sKlotho groups. Numbers (1 to 5 ) represent different animals in a given group. Graphic representations of fibronectin (C) and $\alpha$-SMA (D) expression in different groups as indicated. * $P<0.05$ versus $5 / 6$ NX alone. Scale bar $=$ $50 \mu \mathrm{m}(\mathbf{A}) .5 / 6 \mathrm{NX}, 5 / 6$ nephrectomized mice injected with pcDNA3; 5/6NX+K, 5/6 nephrectomized mice injected with pV5-sKlotho.

(Figure 2, A-E). Consistently, immunostaining revealed that $\beta$-catenin was clearly induced in renal tubular epithelium in remnant kidneys, compared with sham controls (Figure 2F). This activation of $\beta$-catenin was accompanied by renal induction of fibronectin, fibroblast-specific protein 1 (FSP1), and $\alpha$-smooth muscle actin ( $\alpha$-SMA), as demonstrated by Western blot analysis and immunostaining (Figure 2, F-I).

\section{Exogenous Klotho Attenuates Renal Injury in Remnant Kidneys}

To study the role of Klotho depletion in the pathogenesis of kidney lesions, we expressed exogenous Klotho in remnant kidneys in vivo. At 2 weeks after 5/6NX, mice were injected intravenously with the expression vector encoding the secreted form of Klotho (pV5-sKlotho) through a hydrodynamic-based gene delivery approach, as previously reported (Figure 3A). ${ }^{16}$ We found that the expression of exogenous sKlotho reduced levels of albuminuria (Figure 3B) and serum creatinine (Figure 3C). Kidney histology revealed significant morphological lesions characterized by mesangial expansion, glomerular hypertrophy, and tubular interstitial fibrosis at 6 weeks after $5 / 6 \mathrm{NX}$, as shown by periodic acid-Schiff and Masson-Trichrome staining (Figure 3D). However, expression of secreted Klotho largely ameliorated these morphological lesions (Figure 3, D-F).

\section{Exogenous Klotho Suppresses RAS Activation in Vivo}

Intrarenal expression of exogenous Klotho was confirmed by immunostaining and Western blot analysis. Injection of pV5-sKlotho expression vector restored renal Klotho protein expression, as reported previously (Figure 4, A-D). ${ }^{15}$ Notably, induction of renal sKlotho protein appeared to be suboptimal at 7 days after last injection of the pV5-sKlotho plasmid (Figure 3A), because transgene expression by this approach usually occurred at 4 to 12 hours after plasmid injection. ${ }^{32}$ Interestingly, consistent with earlier observation, ${ }^{16}$ expression of sKlotho in vivo was able to induce membranous Klotho expression (Figure 4, B and C), presumably as a result of blocking the TGF- $\beta 1-$ mediated repression of endogenous Klotho expression. 
A
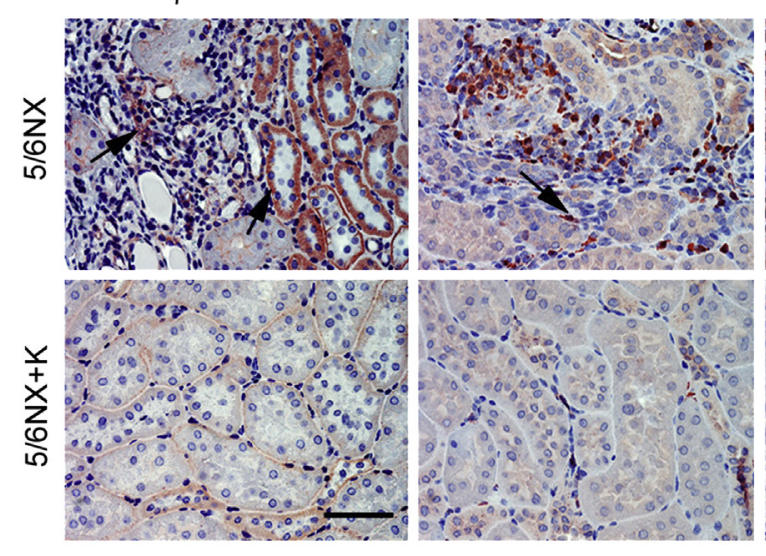

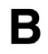

B

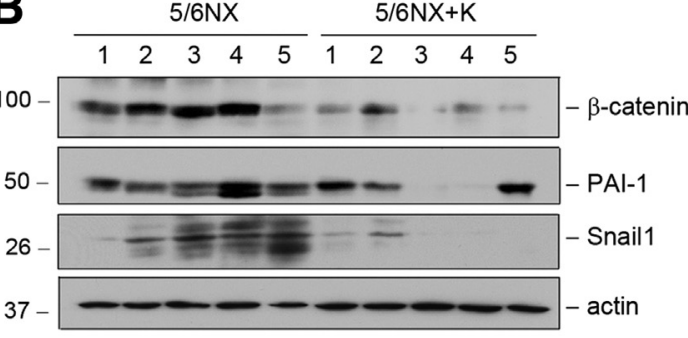

MMP-7
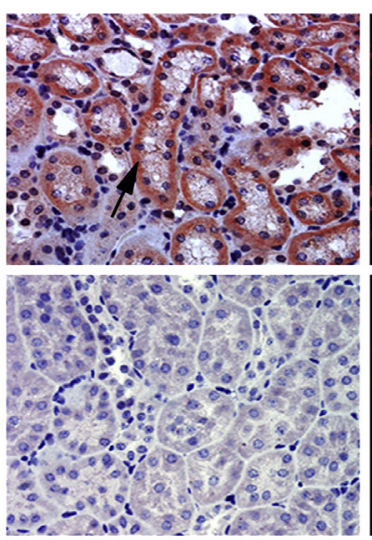

PAI-1

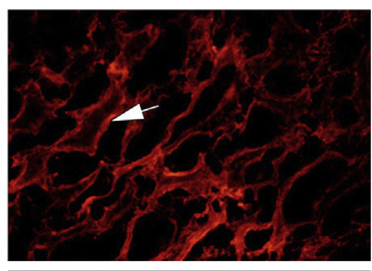

C

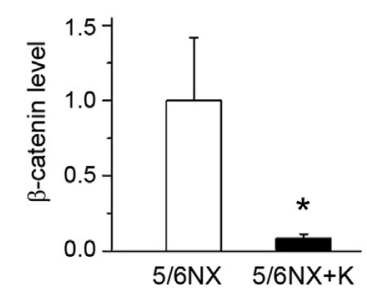

D

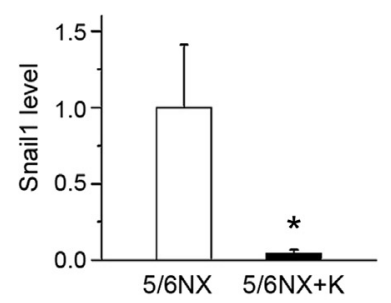

Figure $7 \quad$ Klotho inhibits renal expression of $\beta$-catenin and its target genes in the mouse remnant kidney model. A: Representative micrographs show $\beta$-catenin, FSP-1, MMP-7, and PAI-1 expression in different groups. Paraffin sections were stained with different antibodies. Arrows indicate positive staining. B: Western blot analyses of renal expression of $\beta$-catenin and its target genes in different groups. Numbers (1 to 5 ) represent different animals in a given group. Graphic representations of $\beta$-catenin (C) and Snail1 (D) expression in different groups as indicated. ${ }^{*} P<0.05$ versus $5 / 6 \mathrm{NX}$ alone. Scale bar $=50 \mu \mathrm{m}$ (A). 5/6NX, 5/6 nephrectomized mice injected with pcDNA3; 5/6NX+K,5/6 nephrectomized mice injected with pV5-sKlotho.

Because RAS plays a pivotal role in the pathogenesis of $\mathrm{CKD},{ }^{7,33}$ we then determined the effect of exogenous Klotho on RAS expression in remnant kidneys. Interestingly, exogenous Klotho was able to markedly inhibit the expression of all RAS components (Figure 4A). We also examined RAS proteins by Western blot analyses. Exogenous Klotho dramatically repressed the expression of AGT, renin, ACE, and AT1 in remnant kidneys (Figure 4, E-I). These data demonstrate that Klotho inhibits the expression of multiple RAS components in vivo, thereby alleviating kidney injury.

\section{Exogenous Klotho Normalizes Blood Pressure in Remnant Kidneys}

Consistent with RAS activation in remnant kidneys, elevation of blood pressure is a major pathological feature of mice after $5 /$ $6 \mathrm{NX}$. Therefore, we examined the effects of Klotho on blood pressure regulation in this model. Both systolic blood pressure (SBP) and mean arterial blood pressure (MABP) were significantly elevated at 6 weeks after $5 / 6 \mathrm{NX}$, as measured by non-invasive tail-cuff method (Figure 5). Interestingly, expression of exogenous sKlotho completely normalized both SBP and MABP in remnant kidney model (Figure 5). These data suggest that exogenous Klotho is able to normalize blood pressure after kidney injury, presumably by inhibiting RAS activation in vivo.

\section{Inhibition of RAS by Klotho Represses Fibrogenic Genes}

We next examined the expression of several key fibrosisrelated genes in remnant kidneys after 5/6NX. Renal expression of TGF- $\beta 1$, the key profibrotic factor that is regulated by angiotensin II, was markedly induced at 6 weeks after $5 / 6 \mathrm{NX}$ (Figure 6A). However, in vivo expression of secreted Klotho almost completely abolished its induction (Figure 6A). The expression of $\alpha$-SMA, the molecular signature of renal myofibroblasts, ${ }^{1}$ was also induced after $5 / 6 \mathrm{NX}$, which was abolished by exogenous Klotho as well (Figure 6A). Similarly, expression of major interstitial matrix components including type I collagen and fibronectin was up-regulated in remnant kidneys, and exogenous Klotho abrogated their induction (Figure 6A). Western blotting and quantitative analyses also demonstrated that Klotho largely inhibited the expression of fibronectin and $\alpha$-SMA (Figure 6, B-D).

We further examined the effects of exogenous Klotho on $\mathrm{Wnt} / \beta$-catenin signaling. Expression of exogenous Klotho inhibited renal expression of $\beta$-catenin and its targets FSP1, MMP-7, and PAI-1 in remnant kidneys (Figure 7A). Furthermore, Western blotting revealed that renal expression of $\beta$-catenin and Snaill was markedly inhibited by Klotho (Figure 7, B-D). Expression of PAI-1 also tended to 
A
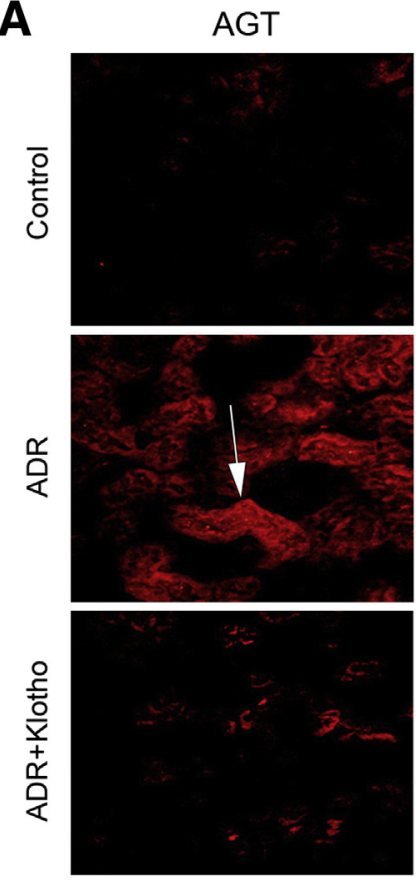

B

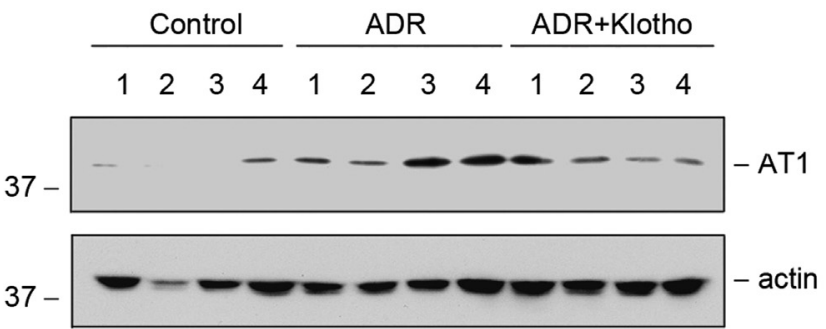

Renin
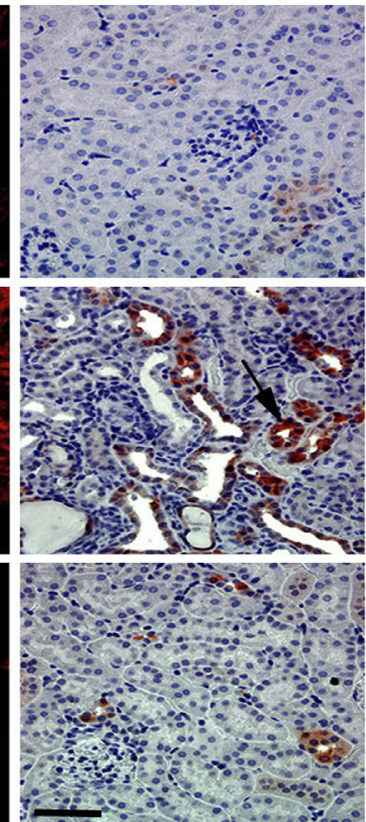

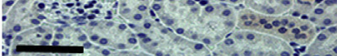

$$
\text { Control } \longrightarrow \text { ADR ADR+Klotho }
$$

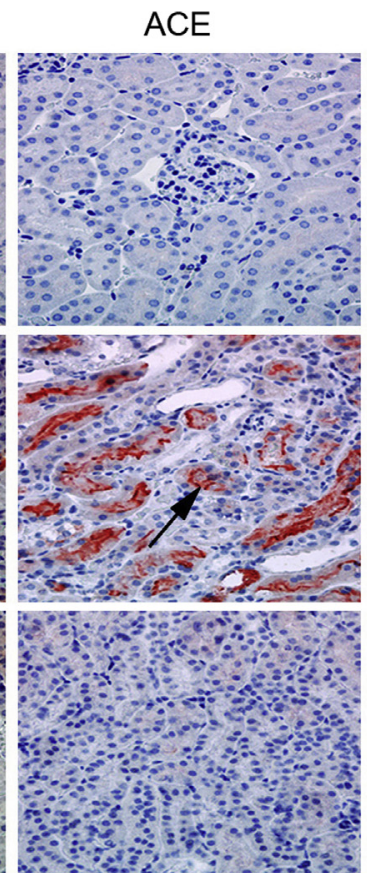

AT1

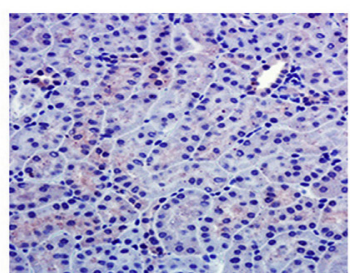

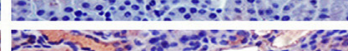
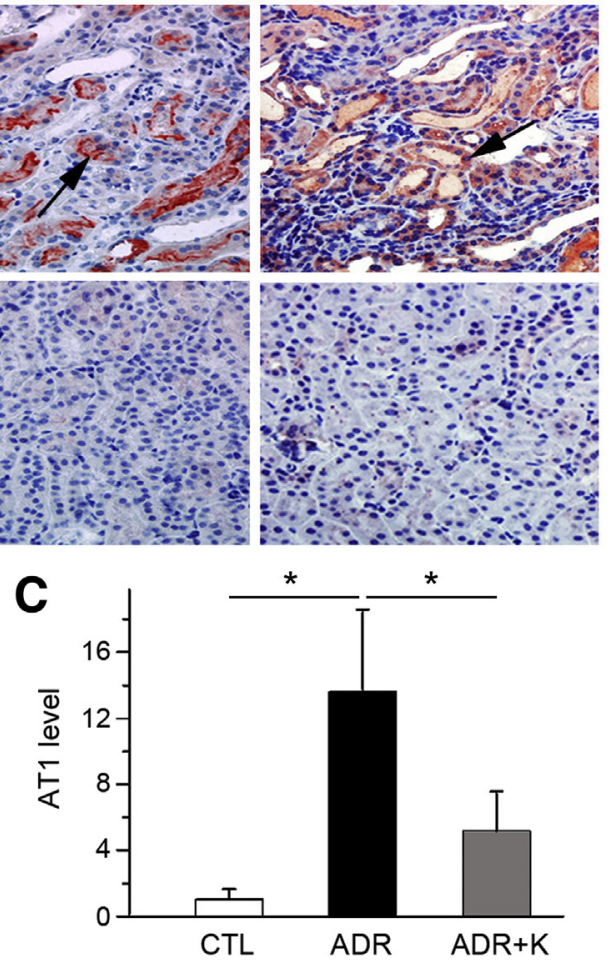

Figure 8 Expression of Klotho inhibits renin-angiotensin system (RAS) activation in adriamycin (ADR) nephropathy. A-C: Representative micrographs show renal expression and localization of RAS components in different groups. Paraffin-embedded or frozen kidney sections were stained with AGT, renin, ACE, and AT1 antibodies. Arrows indicate positive staining. B: Western blot analyses show AT1 expression in different groups as indicated. Numbers (1 to 4) represent different animals in a given group. C: Graphic presentation shows that expression of Klotho inhibits AT1 expression in ADR nephropathy. Relative protein levels over the controls (fold induction) are reported. $n=5$ to 6 (C). ${ }^{*} P<0.05$. Scale bar $=50 \mu \mathrm{m}(\mathbf{A})$. ADR, ADR mice injected with pcDNA3; $A D R+K, A D R$ mice injected with pV5-sKlotho.

be reduced by expression of exogenous Klotho (Figure 7B), although it was not statistically significant (data not shown).

\section{Klotho Inhibits RAS Activation in Adriamycin and Obstructive Nephropathy}

To confirm whether Klotho inhibition of RAS is a generalized phenomenon, we investigated the effect of exogenous Klotho on RAS activation in adriamycin nephropathy and UUO. Mice were injected with pV5-sKlotho plasmid at 1 and 2 weeks after adriamycin injury, as previously reported, ${ }^{16}$ and renal RAS expression was examined at 3 weeks. Delivery of sKlotho clearly blocked AGT, renin, ACE, and AT1 expression in diseased kidneys (Figure 8A). Results were similar when AT1 protein was analyzed by Western blot (Figure 8, B and C).

Klotho was also capable of inhibiting RAS in the UUO model of kidney fibrosis. Mice were injected with
pV5-sKlotho plasmid at 1 day before or 3 days after UUO surgery, as described previously, ${ }^{15}$ and renal RAS expression was examined at 7 days. Immunostaining revealed that all components of RAS, including AGT, renin, ACE, and AT1, were up-regulated in the obstructed kidney, and exogenous Klotho abolished their induction (Figure 9A). We further analyzed renin and AT1 protein by Western blot analyses. Delivery of the secreted form of Klotho repressed renal expression of renin and AT1 in obstructive nephropathy (Figure 9, B and $\mathrm{C}$ ). These data suggest that Klotho elicits renal protection by inhibiting RAS expression in multiple models of CKD.

\section{Klotho Inhibits Wnt-Mediated RAS Activation in Vitro}

To investigate the cause-effect relationship between Klotho depletion and RAS activation, we further examined 
A
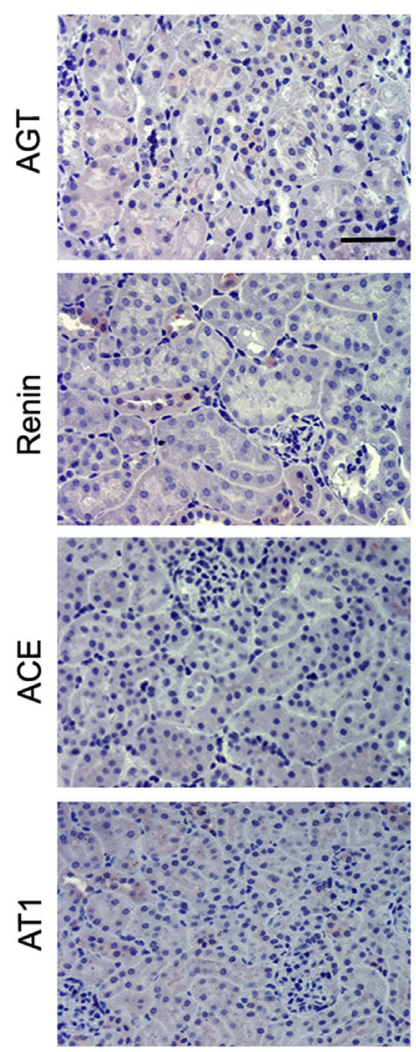

B

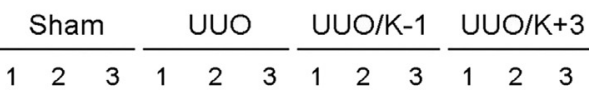
37.
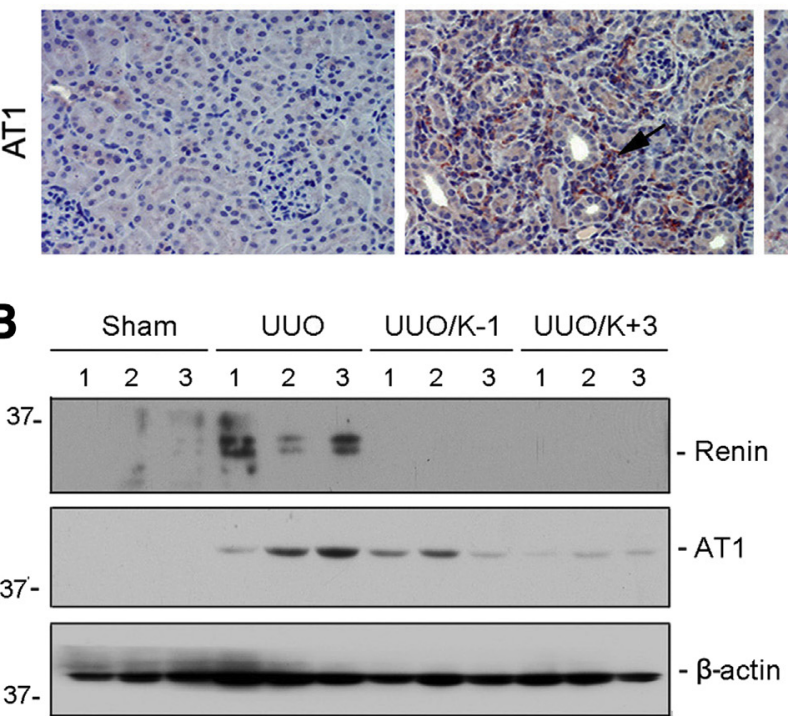

UUO/K-1
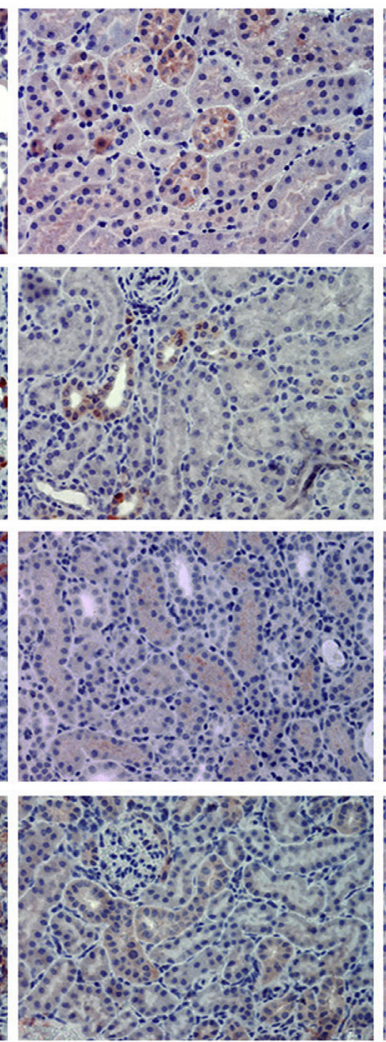

C

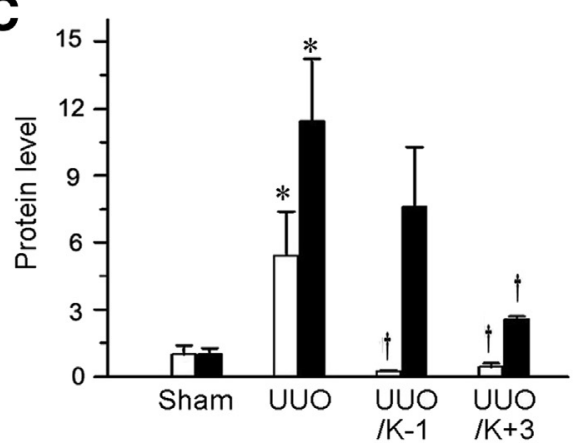

Figure 9 Expression of sKlotho inhibits renin-angiotensin system (RAS) activation in obstructive nephropathy. A: Representative micrographs show renal expression and localization of RAS components in different groups. Kidney sections were stained with AGT, renin, ACE, and AT1 antibodies. Arrows indicate positive staining. B: Western blot analyses show that exogenous sKlotho inhibits renin and AT1 expression in different groups as indicated. Numbers (1 to 3) represent different animals in a given group. C: Graphic presentation show expression of sKlotho inhibits renin (white bars) and AT1 (black bars) expression in unilateral ureteral obstruction (UU0) kidneys. Relative protein levels over the controls (fold-induction) are reported. $n=5$ to 6 (C). ${ }^{*} P<0.05$ versus sham controls; ${ }^{\dagger} P<0.05$ versus UU0. Scale bar $=50 \mu \mathrm{m}(\mathbf{A})$. UU0, UU0 mice injected with pcDNA3; UU0/K-1, UU0 mice injected with pV5-sKlotho 1 day before surgery; UU0/K+3, UU0 mice injected with pV5-sKlotho 3 days after surgery.

the regulation of RAS genes by Klotho in kidney epithelial cells in vitro. To this end, human proximal tubular epithelial cells (HKC-8) were transfected with a hemagglutinin (HA)-tagged Wnt1 expression vector (pHA-Wnt1). As previously reported, ${ }^{16}$ expression of Wnt1 (Figure 10, A-D) significantly induced protein expression of RAS genes in HKC-8 cells. However, co-expression of Klotho dose-dependently repressed the Wnt1-mediated induction of all RAS genes (Figure 10, A-D). There results indicate that Klotho is capable of inhibiting multiple RAS genes in vitro as well.

\section{Discussion}

Intrarenal RAS activation is widely recognized as a major driver promoting the development and progression of CKD, hypertension, and cardiovascular disease. ${ }^{7,34-36}$ In this study, we demonstrate that Klotho, an antiaging protein 

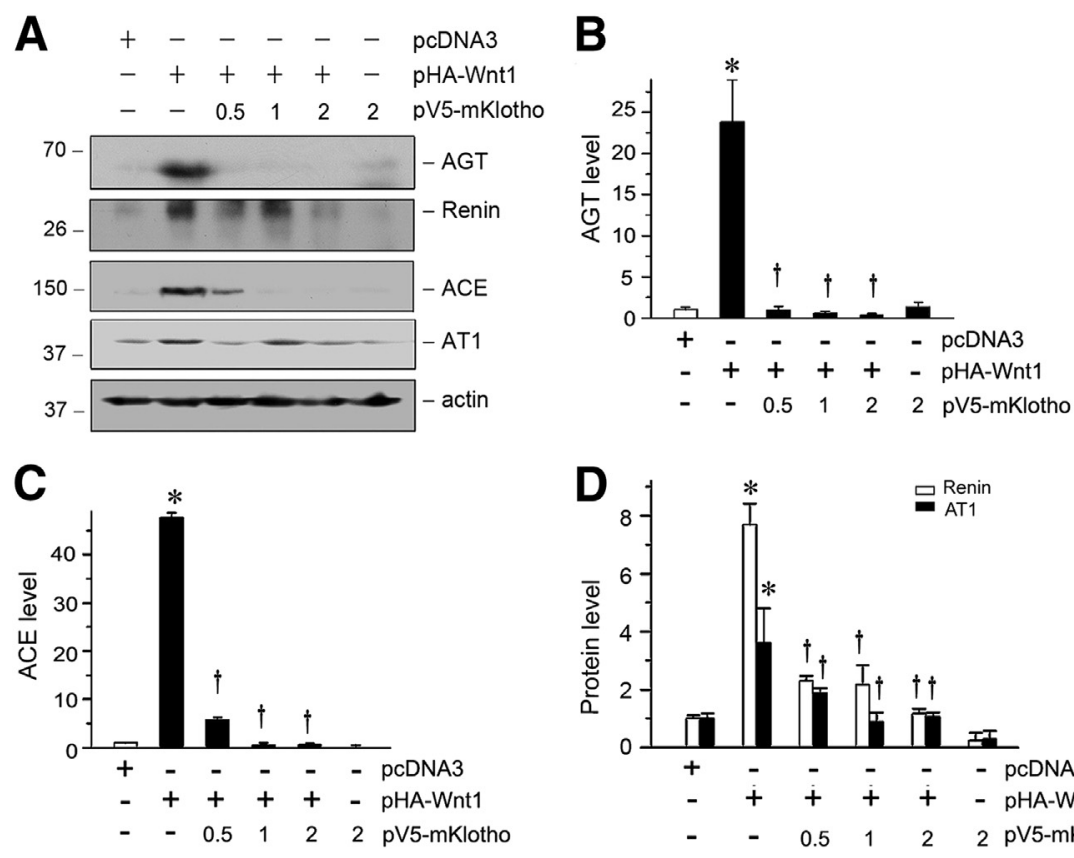

Figure 10 Klotho inhibits Wnt1-mediated renin-angiotensin system (RAS) induction in vitro. A: Klotho dose-dependently inhibits Wnt1mediated induction of RAS components, such as AGT, renin, ACE, and AT1. HKC-8 cells were transfected with Wnt1 expression plasmid (pHAWnt1) and Klotho expression vector (pV5mKlotho) as indicated. Cells lysates were then blotted with antibodies against different RAS components. B-D: Quantitative data show the expression of AGT, ACE, renin, and AT1 in different groups as indicated. ${ }^{*} P<0.05$ versus pcDNA3 alone; ${ }^{\dagger} P<0.05$ versus $\mathrm{pHA}-W n$ t1 alone. which is highly expressed in renal tubular epithelium of normal adult kidneys, inhibits renal expression of all RAS components. Expression of exogenous Klotho effectively blocks RAS activation and ameliorates kidney injury in three models of CKD induced by $5 / 6 \mathrm{NX}$, adriamycin, and UUO. Klotho also normalizes blood pressure in remnant kidneys, a widely used model characterized by hypertension. In vitro, Klotho abolishes the Wnt ligand-mediated induction of RAS components in kidney tubular epithelial cells. These results provide convincing evidence for an intrinsic linkage between Klotho depletion and RAS activation in aged kidneys and in CKD. Our studies also offer significant insights into the mechanism by which Klotho exerts its renal protection.

Hyperactive RAS, through its active component angiotensin II, promotes kidney injury by a variety of mechanisms. In addition to causing hypertension, angiotensin II has been demonstrated to induce TGF- $\beta$ expression, promote the generation of reactive oxygen species, and augment renal inflammation, all of which are imperative in the initiation and progression of CKD. ${ }^{8,33}$ Extensive studies indicate that local activation of intrarenal RAS, rather than its systemic levels, dictates tissue damage in the kidneys. ${ }^{9,35,37,38}$ Although the importance of intrarenal RAS activation in the evolution of CKD is well established, how RAS components are regulated under pathological conditions remained incompletely understood. In this context, the observations that Klotho represses the expression of all RAS proteins both in vivo and in vitro are quite significant. This finding is supported by several lines of evidence: i) loss of Klotho is associated with RAS activation in the diseased kidneys; ii) in vivo expression of exogenous Klotho inhibits the expression of all RAS proteins and normalizes blood pressure; and iii) Klotho also represses RAS induction in kidney tubular epithelial cells in vitro. Taken together, these results suggest that abundant Klotho protein in normal kidneys would prevent RAS activation, a previously unrecognized and important function of this antiaging protein.

Klotho is highly expressed in young and healthy animals primarily in kidney tubular epithelium, and its expression is spontaneously down-regulated in aged animals and in the elderly population. ${ }^{13}$ It is of interest to point out that aging is an independent and strong predictor for developing CKD, and aging-related nephropathy is characterized by progressive function decline, hypertension, and kidney fibrosis, features similar to CKD occurring by various etiologies. ${ }^{4,6}$ This is not surprising because loss of Klotho is a common feature in both aging and CKD. Of note, such a loss of Klotho in aging and CKD is probably mediated by increased renal TGF- $\beta 1^{16}$ and/or angiotensin II. ${ }^{39}$ Consistent with this view, extensive studies show that RAS blockade with various strategies is associated with up-regulation of Klotho expression. ${ }^{40-44}$ Our current studies may also provide new explanation of some earlier observations. For instance, Mitani et $\mathrm{al}^{39}$ reported that in vivo expression of exogenous Klotho prevented long-term angiotensin II infusion-induced renal damage, which could have resulted from a potential depression of up-regulated expression of AT1 and renin, rendering angiotensin II relatively ineffective.

Aging is also associated with other conditions, including increased incidence of hypertension and cardiovascular disorders. The mechanisms underlying how aging leads to hypertension, CKD, and cardiovascular disorders, however, remain poorly understood. Our results on Klotho inhibition of RAS activation in the present study may explain, at least partially, this phenomenon. Of interest, exogenous Klotho also normalizes blood pressure in $5 / 6 \mathrm{NX}$ mice (Figure 5), suggesting that the beneficial effect of Klotho can be 
partially mediated via blood pressure reduction, which is also relevant to RAS inhibition. Notably, a recent study has implicated Klotho deficiency in causing salt-sensitive hypertension in mice, ${ }^{20}$ supporting the role of Klotho in regulating blood pressure. In view of the fact that RAS activation, not only plays a role in the evolution and progression of CKD, but also is a causative factor for developing hypertension and cardiovascular diseases, ${ }^{7,45}$ our studies on the intimate connection between loss of Klotho and RAS activation would have wide implications beyond CKD.

The linkage between Klotho and RAS appears to be mediated by $\mathrm{Wnt} / \beta$-catenin signaling, a pathway that plays a fundamental role in kidney development and injury repair. ${ }^{46-49}$ Despite being relatively silent in normal adult kidneys, $\mathrm{Wnt} / \beta$-catenin is re-activated in a wide variety of CKD such as obstructive nephropathy, diabetic nephropathy, adriamycin nephropathy, polycystic kidney disease, and chronic allograft nephropathy. ${ }^{24,25,50-52}$ We previously reported that Klotho is an endogenous Wnt antagonist through binding and sequestration of Wnt ligands, and therefore loss of Klotho in CKD would result in de-repression of $\mathrm{Wnt} / \beta$-catenin, leading to the induction of its target genes. ${ }^{16}$ Interestingly, multiple RAS genes are novel targets of $\mathrm{Wnt} / \beta$-catenin signaling, and blockade of $\beta$-catenin signaling by a small-molecule inhibitor is able to inhibit RAS and ameliorate kidney injury. ${ }^{12}$ In harmony with this notion, renal $\beta$-catenin and its downstream target proteins such as Snail1, PAI-1, and MMP-7 are upregulated in diseased kidneys, ${ }^{23,28,31,51,52}$ which is concurrent with RAS activation. Therefore, Wnt/ $\beta$-catenin presumably functions as a mediator in coupling Klotho depletion with RAS activation in various CKD including aging-related nephropathy.

The mainstay of current therapy for CKD clinically is to use RAS inhibitors, including ACE inhibitors and AT1 receptor blockers. ${ }^{33}$ However, these approaches often lead to compensatory renin up-regulation in the kidneys. Studies show that renin not only enzymatically converts AGT to angiotensin I but also binds to the (pro)renin receptor, which triggers a cascade of signaling events that lead to induction of fibrosis-related proteins such as fibronectin. ${ }^{34,53}$ Therefore, strategies to repress all RAS expression, rather than their activities, would be more effective for the treatment of CKD. In this context, restoration of endogenous Klotho expression or supplementation with exogenous Klotho could be a feasible strategy for therapeutic intervention of CKD by inhibiting the expression of all RAS genes.

In summary, we have shown in this study that loss of Klotho leads to induction of all RAS genes, suggesting that this antiaging protein possesses a novel function in the kidneys by constraining RAS activation. Although more studies are needed, our findings suggest that exogenous Klotho may hold promise as a therapeutic agent for CKD by targeted inhibition of multiple RAS genes.

\section{Acknowledgment}

We thank Dr. Lorraine C. Rascusen (Johns Hopkins University, Baltimore, MD) for the gift of the HKC clone-8.

\section{References}

1. Liu Y: Cellular and molecular mechanisms of renal fibrosis. Nat Rev Nephrol 2011, 7:684-696

2. Zhang L, Wang F, Wang L, Wang W, Liu B, Liu J, Chen M, He Q, Liao Y, Yu X, Chen N, Zhang JE, Hu Z, Liu F, Hong D, Ma L, Liu H, Zhou X, Chen J, Pan L, Chen W, Li X, Wang H: Prevalence of chronic kidney disease in China: a cross-sectional survey. Lancet 2012, 379:815-822

3. McClellan AC, Plantinga L, McCellan WM: Epidemiology, geography and chronic kidney disease. Curr Opin Nephrol Hypertens 2012. 21:323-328

4. Perico N, Remuzzi G, Benigni A: Aging and the kidney. Curr Opin Nephrol Hypertens 2011, 20:312-317

5. Bidani AK, Polichnowski AJ, Loutzenhiser R, Griffin KA: Renal microvascular dysfunction, hypertension and CKD progression. Curr Opin Nephrol Hypertens 2013, 22:1-9

6. Zhou XJ, Rakheja D, Yu X, Saxena R, Vaziri ND, Silva FG: The aging kidney. Kidney Int 2008, 74:710-720

7. Santos PC, Krieger JE, Pereira AC: Renin-angiotensin system, hy pertension, and chronic kidney disease: pharmacogenetic implications. J Pharmacol Sci 2012, 120:77-88

8. Crowley SD, Coffman TM: Recent advances involving the reninangiotensin system. Exp Cell Res 2012, 318:1049-1056

9. Graciano ML, Cavaglieri RdeC, Delle H, Dominguez WV, Casarini DE, Malheiros DM, Noronha IL: Intrarenal reninangiotensin system is upregulated in experimental model of progressive renal disease induced by chronic inhibition of nitric oxide synthesis. J Am Soc Nephrol 2004, 15:1805-1815

10. Cao W, Xu J, Zhou ZM, Wang GB, Hou FF, Nie J: Advanced oxidation protein products activate intrarenal renin-angiotensin system via a CD36-mediated, redox-dependent pathway. Antioxid Redox Signal 2013, 18:19-35

11. Cao W, Zhou QG, Nie J, Wang GB, Liu Y, Zhou ZM, Hou FF: Albumin overload activates intrarenal renin-angiotensin system through protein kinase $\mathrm{C}$ and NADPH oxidase-dependent pathway. J Hypertens 2011, 29:1411-1421

12. Zhou L, Li Y, Hao S, Zhou D, Tan RJ, Nie J, Hou FF, Kahn M, Liu Y: Multiple genes of the renin-angiotensin system are novel targets of Wnt/ $\beta$-catenin signaling. J Am Soc Nephrol 2015, 26: $107-120$

13. Hu MC, Kuro-o M, Moe OW: Klotho and chronic kidney disease. Contrib Nephrol 2013, 180:47-63

14. Kuro-o M: Klotho and aging. Biochim Biophys Acta 2009, 1790 1049-1058

15. Kuro-o M: Klotho and betaKlotho. Adv Exp Med Biol 2012, 728: $25-40$

16. Zhou L, Li Y, Zhou D, Tan RJ, Liu Y: Loss of Klotho contributes to kidney injury by derepression of Wnt/ $\beta$-catenin signaling. J Am Soc Nephrol 2013, 24:771-785

17. Zhao Y, Banerjee S, Dey N, LeJeune WS, Sarkar PS, Brobey R, Rosenblatt KP, Tilton RG, Choudhary S: Klotho depletion contributes to increased inflammation in kidney of the $\mathrm{db} / \mathrm{db}$ mouse model of diabetes via RelA (serine)536 phosphorylation. Diabetes 2011, 60: 1907-1916

18. Yang HC, Zuo Y, Fogo AB: Models of chronic kidney disease. Drug Discov Today Dis Models 2010, 7:13-19

19. Leelahavanichkul A, Yan Q, Hu X, Eisner C, Huang Y, Chen R, Mizel D, Zhou H, Wright EC, Kopp JB, Schnermann J, Yuen PS, Star RA: Angiotensin II overcomes strain-dependent resistance of 
rapid CKD progression in a new remnant kidney mouse model. Kidney Int 2010, 78:1136-1153

20. Zhou X, Chen K, Lei H, Sun Z: Klotho gene deficiency causes saltsensitive hypertension via monocyte chemotactic protein-1/CC chemokine receptor 2-mediated inflammation. J Am Soc Nephrol 2015, 26:121-132

21. Feng M, Whitesall S, Zhang Y, Beibel M, D'Alecy L, DiPetrillo K: Validation of volume-pressure recording tail-cuff blood pressure measurements. Am J Hypertens 2008, 21:1288-1291

22. Whitesall SE, Hoff JB, Vollmer AP, D'Alecy LG: Comparison of simultaneous measurement of mouse systolic arterial blood pressure by radiotelemetry and tail-cuff methods. Am J Physiol Heart Circ Physiol 2004, 286:H2408-H2415

23. Dai C, Stolz DB, Kiss LP, Monga SP, Holzman LB, Liu Y: Wnt/ßcatenin signaling promotes podocyte dysfunction and albuminuria. J Am Soc Nephrol 2009, 20:1997-2008

24. He W, Kang YS, Dai C, Liu Y: Blockade of Wnt/ß-catenin signaling by paricalcitol ameliorates proteinuria and kidney injury. J Am Soc Nephrol 2011, 22:90-103

25. He W, Dai C, Li Y, Zeng G, Monga SP, Liu Y: Wnt/ß-catenin signaling promotes renal interstitial fibrosis. J Am Soc Nephrol 2009, 20:765-776

26. Raij L, Azar S, Keane W: Mesangial immune injury, hypertension, and progressive glomerular damage in Dahl rats. Kidney Int 1984, 26 : $137-143$

27. Ots M, Mackenzie HS, Troy JL, Rennke HG, Brenner BM: Effects of combination therapy with enalapril and losartan on the rate of progression of renal injury in rats with 5/6 renal mass ablation. J Am Soc Nephrol 1998, 9:224-230

28. He W, Tan RJ, Li Y, Wang D, Nie J, Hou FF, Liu Y: Matrix metalloproteinase-7 as a surrogate marker predicts renal $\mathrm{Wnt} / \beta$-catenin activity in CKD. J Am Soc Nephrol 2012, 23:294-304

29. Zhou D, Li Y, Zhou L, Tan RJ, Xiao LM, Liang M, Hou FF, Liu Y: Sonic hedgehog is a novel tubule-derived growth factor for interstitial fibroblasts after kidney injury. J Am Soc Nephrol 2014, 25: $2187-2200$

30. Liu H, Fergusson MM, Castilho RM, Liu J, Cao L, Chen J, Malide D, Rovira II, Schimel D, Kuo CJ, Gutkind JS, Hwang PM, Finkel T: Augmented Wnt signaling in a mammalian model of accelerated aging. Science 2007, 317:803-806

31. He W, Tan R, Dai C, Li Y, Wang D, Hao S, Kahn M, Liu Y: Plasminogen activator inhibitor- 1 is a transcriptional target of the canonical pathway of Wnt/ß-catenin signaling. J Biol Chem 2010, 285:24665-24675

32. Dai C, Yang J, Bastacky S, Xia J, Li Y, Liu Y: Intravenous administration of hepatocyte growth factor gene ameliorates diabetic nephropathy in mice. J Am Soc Nephrol 2004, 15:2637-2647

33. Ruggenenti P, Cravedi P, Remuzzi G: Mechanisms and treatment of CKD. J Am Soc Nephrol 2012, 23:1917-1928

34. Nguyen G: Renin and prorenin receptor in hypertension: what's new? Curr Hypertens Rep 2011, 13:79-85

35. Gurley SB, Riquier-Brison AD, Schnermann J, Sparks MA, Allen AM, Haase VH, Snouwaert JN, Le TH, McDonough AA, Koller BH, Coffman TM: AT1A angiotensin receptors in the renal proximal tubule regulate blood pressure. Cell Metab 2011, 13:469-475

36. Burns WC, Thomas MC: Angiotensin II and its role in tubular epithelial to mesenchymal transition associated with chronic kidney disease. Cells Tissues Organs 2011, 193:74-84
37. Kobori H, Nangaku M, Navar LG, Nishiyama A: The intrarenal renin-angiotensin system: from physiology to the pathobiology of hypertension and kidney disease. Pharmacol Rev 2007, 59:251-287

38. Crowley SD, Gurley SB, Herrera MJ, Ruiz P, Griffiths R, Kumar AP, Kim HS, Smithies O, Le TH, Coffman TM: Angiotensin II causes hypertension and cardiac hypertrophy through its receptors in the kidney. Proc Natl Acad Sci U S A 2006, 103:17985-17990

39. Mitani H, Ishizaka N, Aizawa T, Ohno M, Usui S, Suzuki T, Amaki T, Mori I, Nakamura Y, Sato M, Nangaku M, Hirata Y, Nagai R: In vivo klotho gene transfer ameliorates angiotensin IIinduced renal damage. Hypertension 2002, 39:838-843

40. Yoon HE, Ghee JY, Piao S, Song JH, Han DH, Kim S, Ohashi N, Kobori H, Kuro-o M, Yang CW: Angiotensin II blockade upregulates the expression of Klotho, the anti-ageing gene, in an experimental model of chronic cyclosporine nephropathy. Nephrol Dial Transplant 2011, 26:800-813

41. Zhou Z, Hu CP, Wang CJ, Li TT, Peng J, Li YJ: Calcitonin generelated peptide inhibits angiotensin II-induced endothelial progenitor cells senescence through up-regulation of klotho expression. Atherosclerosis 2010, 213:92-101

42. Zhou Q, Lin S, Tang R, Veeraragoo P, Peng W, Wu R: Role of fosinopril and valsartan on klotho gene expression induced by angiotensin II in rat renal tubular epithelial cells. Kidney Blood Press Res 2010, 33:186-192

43. Tang R, Zhou QL, Ao X, Peng WS, Veeraragoo P, Tang TF: Fosinopril and losartan regulate klotho gene and nicotinamide adenine dinucleotide phosphate oxidase expression in kidneys of spontaneously hypertensive rats. Kidney Blood Press Res 2011, 34:350-357

44. Zanchi C, Locatelli M, Benigni A, Corna D, Tomasoni S, Rottoli D, Gaspari F, Remuzzi G, Zoja C: Renal expression of FGF23 in progressive renal disease of diabetes and the effect of ACE inhibitor. PLoS One 2013, 8:e70775

45. Herzog CA, Asinger RW, Berger AK, Charytan DM, Diez J, Hart RG, Eckardt KU, Kasiske BL, McCullough PA, Passman RS, DeLoach SS, Pun PH, Ritz E: Cardiovascular disease in chronic kidney disease. A clinical update from Kidney Disease: improving Global Outcomes (KDIGO). Kidney Int 2011, 80:572-586

46. Angers S, Moon RT: Proximal events in Wnt signal transduction. Nat Rev Mol Cell Biol 2009, 10:468-477

47. Yu J, Carroll TJ, Rajagopal J, Kobayashi A, Ren Q, McMahon AP: A Wnt7b-dependent pathway regulates the orientation of epithelial cell division and establishes the cortico-medullary axis of the mammalian kidney. Development 2009, 136:161-171

48. Wang D, Dai C, Li Y, Liu Y: Canonical Wnt/ß-catenin signaling mediates transforming growth factor- $\beta 1$-driven podocyte injury and proteinuria. Kidney Int 2011, 80:1159-1169

49. Clevers H, Nusse R: Wnt/ß-catenin signaling and disease. Cell 2012, 149:1192-1205

50. Zhou T, He X, Cheng R, Zhang B, Zhang RR, Chen Y, Takahashi Y, Murray AR, Lee K, Gao G, Ma JX: Implication of dysregulation of the canonical wingless-type MMTV integration site (WNT) pathway in diabetic nephropathy. Diabetologia 2012, 55:255-266

51. Zhou L, Liu Y: Wnt/ß-catenin signalling and podocyte dysfunction in proteinuric kidney disease. Nat Rev Nephrol 2015, 11:535-545

52. Tan RJ, Zhou D, Zhou L, Liu Y: Wnt/ß-catenin signaling and kidney fibrosis. Kidney Int Suppl 2014, 4:84-90

53. Nguyen G, Muller DN: The biology of the (pro)renin receptor. J Am Soc Nephrol 2010, 21:18-23 